\title{
Gravity/Topography Transfer Function and Isostatic Geoid Anomalies
}

(Copyright 2002, David T. Sandwell)

This lecture combines thin-elastic plate flexure theory with the solution to Poisson's equation to develop a linear relationship between gravity and topography. This relationship can be used in a variety of ways.

(1) If both the topography and gravity are measured over an area that is several times greater then the flexural wavelength, then the gravity/topography relationship (in the wavenumber domain) can be used to estimate the elastic thickness of the lithosphere and/or the crustal thickness. There are many good references on this topic including Dorman and Lewis [1972], McKenzie and Bowin, [1976]; Banks et al., [1977]; Watts, [1978]; McNutt, [1979].

(2) At wavelengths greater than the flexural wavelength where features are isostaticallycompensated, the geoid/topography ratio can be used to estimate the depth of compensation of crustal plateaus and the depth of compensation of hot-spot swells [Haxby and Turcotte, 1978].

(3) If the gravity field is known over a large area but there is rather sparse ship-track coverage, the topography/gravity transfer function can be used to interpolate the seafloor depth among the sparse ship soundings [Smith and Sandwell, 1994].

\section{Flexure theory}

In a previous lecture we developed an analytic solution for the response of a thin-elastic plate floating on a fluid mantle that is subjected to a line load. Here we follow the same approach but solve the flexure equation for an arbitrary vertical load representing, for example, the loading of the lithosphere due to the weight of a volcano as shown in the following diagram

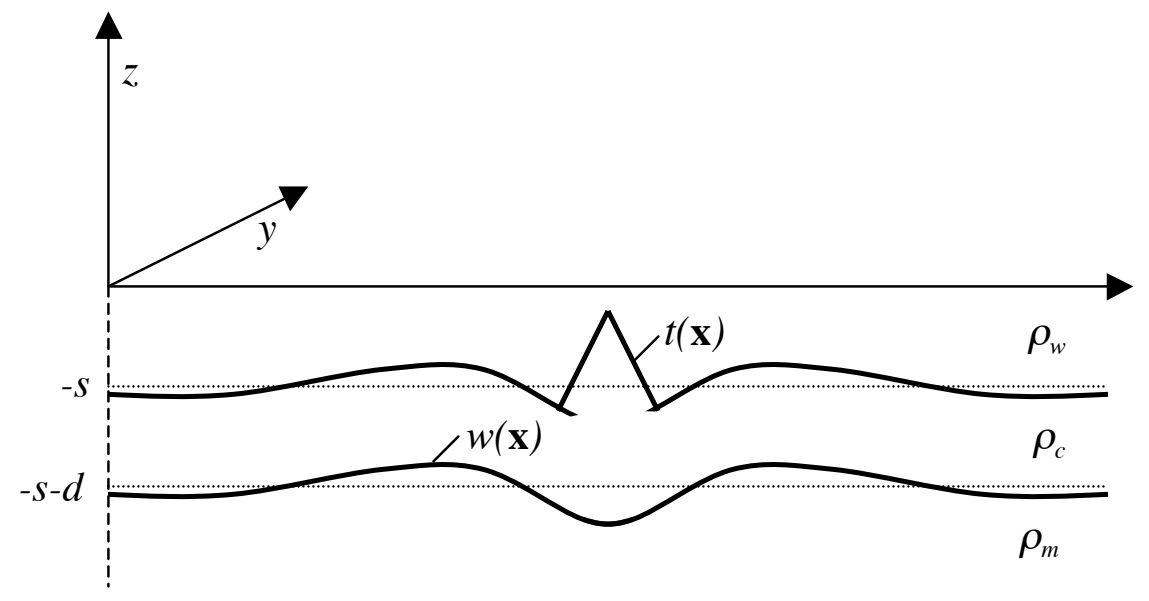

where $s$ is the mean ocean depth $(\sim 4 \mathrm{~km})$ and $d$ is the thickness of the crust $(\sim 6 \mathrm{~km})$. The topography of the Moho is equal to deflection of the elastic plate $w(\mathbf{x})$. The topography of the seafloor, $t(\mathbf{x})$, has two components; the topographic load, $t_{o}(\mathbf{x})$, and the deflection of the elastic plate $w(\mathbf{x})$. 
$t(\mathbf{x})=t_{o}(\mathbf{x})+w(\mathbf{x})$

For this calculation, we make the following assumptions: the thickness of the elastic plate is less than the flexural wavelength; the deflection of the elastic plate is much less than the flexural wavelength; the flexural rigidity, $D$, is constant; and there is no end-load on the plate so $F=0$. The vertical force balance for flexure of a thin elastic plate floating on the mantle is described by the following differential equation

$$
D\left(\frac{\partial^{4}}{\partial x^{4}}+2 \frac{\partial^{4}}{\partial x^{2} \partial y^{2}}+\frac{\partial^{4}}{\partial y^{4}}\right) w(\mathbf{x})+\left(\rho_{m}-\rho_{w}\right) g w(\mathbf{x})=-\left(\rho_{c}-\rho_{w}\right) g t_{o}(\mathbf{x})
$$

where the parameters are defined in the following table.

\begin{tabular}{|c|l|c|}
\hline Parameter & Definition & Value/Unit \\
\hline$w(\mathbf{x})$ & $\begin{array}{l}\text { deflection of plate } \\
\text { (positive up) }\end{array}$ & $\mathrm{m}$ \\
\hline$D=\frac{E h^{3}}{12(1-v)}$ & flexural rigidity & $\mathrm{N} \mathrm{m}$ \\
\hline$h$ & elastic plate thickness & $1025 \mathrm{~kg} \mathrm{~m}^{-3}$ \\
\hline$\rho_{w}$ & seawater density & $2800 \mathrm{~kg} \mathrm{~m}^{-3}$ \\
\hline$\rho_{c}$ & seawater density & $3330 \mathrm{~kg} \mathrm{~m}^{-3}$ \\
\hline$\rho_{m}$ & mantle density & $9.82 \mathrm{~m} \mathrm{~s}^{-2}$ \\
\hline$g$ & acceleration of gravity & $6.5 \times 10^{10} \mathrm{~Pa}^{-}$ \\
\hline$E$ & Young's modulus & 0.25 \\
\hline$v$ & Poisson's ratio & \\
\hline
\end{tabular}

Take the 2-D fourier transform of (2) to reduce the differential equation to an algebraic equation,

$$
D(2 \pi)^{4}\left(k_{x}^{4}+2 k_{x}^{2} k_{y}^{2}+k_{y}^{4}\right) W(\mathbf{k})+\left(\rho_{m}-\rho_{w}\right) g W(\mathbf{k})=-\left(\rho_{c}-\rho_{w}\right) g[T(\mathbf{k})-W(\mathbf{k})]
$$

where we have used equation (1) to replace $T_{o}(\mathbf{k})$. With a little algebra and noting that $|\mathbf{k}|^{4}=\left(k_{x}^{2}+k_{y}^{2}\right)^{2}$ this can be re-written as

$$
D(2 \pi|\mathbf{k}|)^{4} W(\mathbf{k})+\left(\rho_{m}-\rho_{c}\right) g W(\mathbf{k})=-\left(\rho_{c}-\rho_{w}\right) g T(\mathbf{k})
$$

Now one can solve for the deflection of the elastic plate in terms of the observed topography 
$W(\mathbf{k})=\frac{-\left(\rho_{c}-\rho_{w}\right)}{\left(\rho_{m}-\rho_{c}\right)}\left[1+\frac{D(2 \pi|\mathbf{k}|)^{4}}{g\left(\rho_{m}-\rho_{c}\right)}\right]^{-1} T(\mathbf{k})$

This equation is called the isostatic response function because it describes the topography of the Moho in terms of the topography of the seafloor. Define the flexural wavelength

$\lambda_{f}=2 \pi\left[\frac{D}{g\left(\rho_{m}-\rho_{c}\right)}\right]^{1 / 4}=\sqrt{2} \pi \alpha$

(Note $\alpha$ is the flexural parameter from a previous lecture.) When the wavelength of the topography is much greater than the flexural wavelength, then the topography of the Moho follows the Airy-compenstion model; this is compensated topography.

$W(\mathbf{k})=\frac{-\left(\rho_{c}-\rho_{w}\right)}{\left(\rho_{m}-\rho_{c}\right)} T(\mathbf{k})$

In contrast, when the wavelength of the topography is much less than the flexural wavelength, the topography of the Moho is zero; this is uncompensated topography. The gravity field of the earth is very sensitive to the degree of isostatic compensation so it is useful to develop the gravity field for this model.

\section{Gravity/topography transfer function}

The gravity anomaly for this model is approximated by compressing the topography into a sheet mass where the surface density is $\left(\rho_{c}-\rho_{w}\right) t(\mathbf{x})$. Similarly the Moho topography is compressed into a sheet mass with surface density $\left(\rho_{m}-\rho_{c}\right) w(\mathbf{x})$. Finally the gravity anomaly in each layer is upward-continued to the ocean surface.

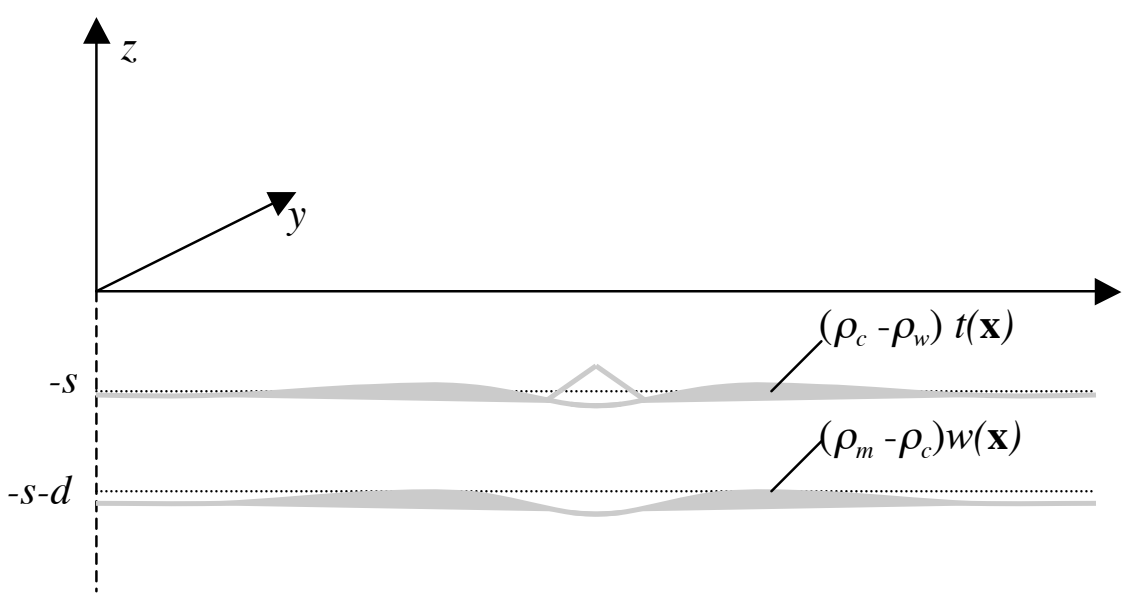


The solution to Poisson's equation (equation 13 in the previous lecture) provides an approximate method of constructing a gravity model for the combined model.

$$
\Delta g(\mathbf{k})=2 \pi G\left(\rho_{c}-\rho_{w}\right) e^{-2 \pi|\mathbf{k}| s} T(\mathbf{k})+2 \pi G\left(\rho_{m}-\rho_{c}\right) e^{-2 \pi|\mathbf{k}|(s+d)} W(\mathbf{k})
$$

Using equation 5, this can be re-written in terms of the observed topography

$$
\Delta g(\mathbf{k})=2 \pi G\left(\rho_{c}-\rho_{w}\right) e^{-2 \pi|\mathbf{k}| s}\left\{1-\left[1+\frac{D(2 \pi|\mathbf{k}|)^{4}}{g\left(\rho_{m}-\rho_{c}\right)}\right]^{-1} e^{-2 \pi|\mathbf{k}| d}\right\} T(\mathbf{k})
$$

This formulation provides a direct approach to constructing gravity anomaly models from seafloor topography: i) take the 2-D fourier transform of the topography; ii) multiply by the gravity-to-topography transfer function; and iii) take the inverse fourier transform of the result. The most important parameter is the elastic plate thickness that is used to estimate the flexural rigidity. The figure below shows the gravity/topography transfer function for a range of elastic thicknesses.

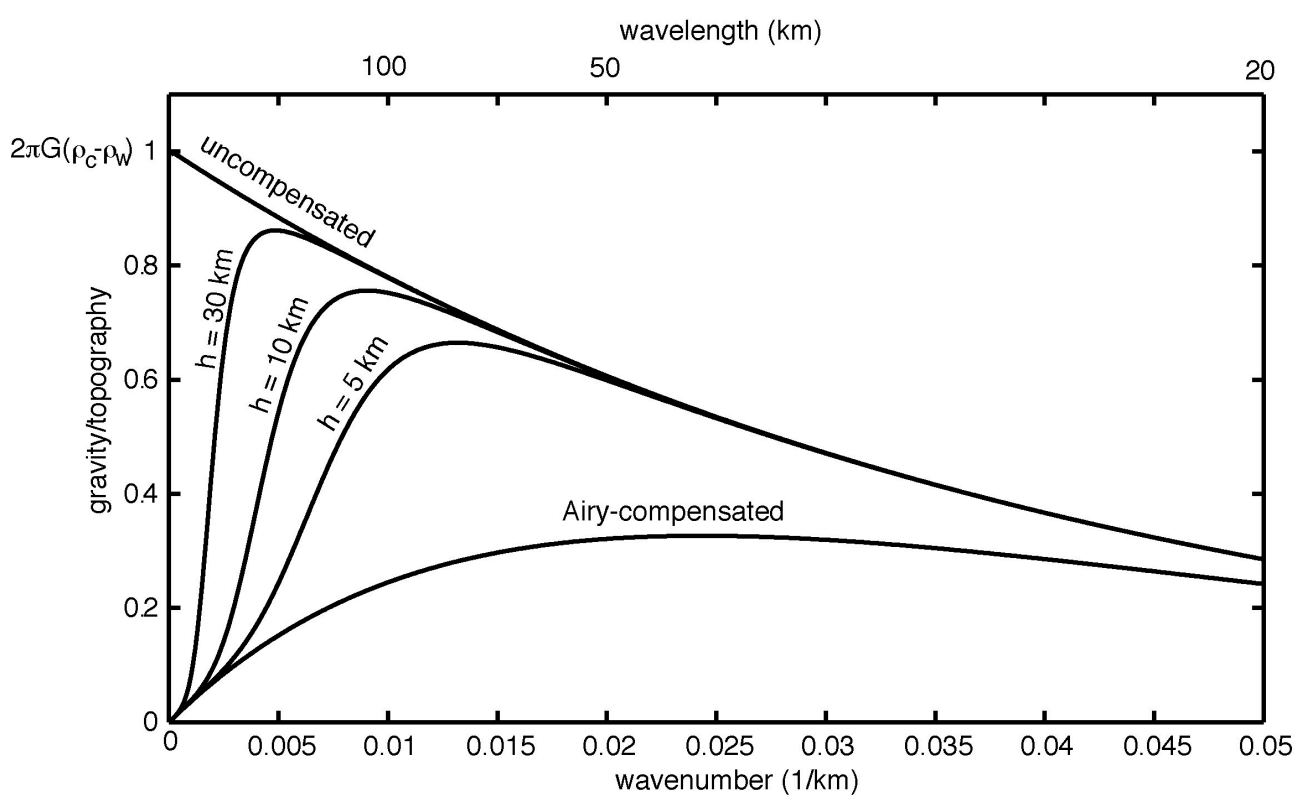

Since the asthenosphere relieves stresses on geological timescales, there is no trulyuncompensated topography. Thus the gravity anomaly for very large-scale structures such as continents and hot-spot swells is small or zero far from the edges of these features. It is only the sharp topographic features such as large seamounts that will have prominent gravity expressions. 
The homework problem is to take a topography profile that crosses the Hawaiian Ridge, construct a model gravity profile for a variety of elastic thicknesses, and compare the result with the observed gravity.

\section{Geoid/topography transfer function}

Using the formulas for converting between geoid and gravity (derived in the lecture on Laplace's equation) it is a simple matter to develop the geoid/topography transfer function.

$$
\begin{aligned}
& N(\mathbf{k})=\frac{1}{2 \pi|\mathbf{k}| g} \Delta g(\mathbf{k}) \\
& \frac{N(\mathbf{k})}{T(\mathbf{k})}=\frac{G\left(\rho_{c}-\rho_{w}\right)}{|\mathbf{k}| g} e^{-2 \pi|\mathbf{k}| s}\left\{1-\left[1+\frac{D(2 \pi|\mathbf{k}|)^{4}}{g\left(\rho_{m}-\rho_{c}\right)}\right]^{-1} e^{-2 \pi|\mathbf{k}| d}\right\} .
\end{aligned}
$$

This geoid topography transfer function has some interesting properties as illustrated in the figure below.

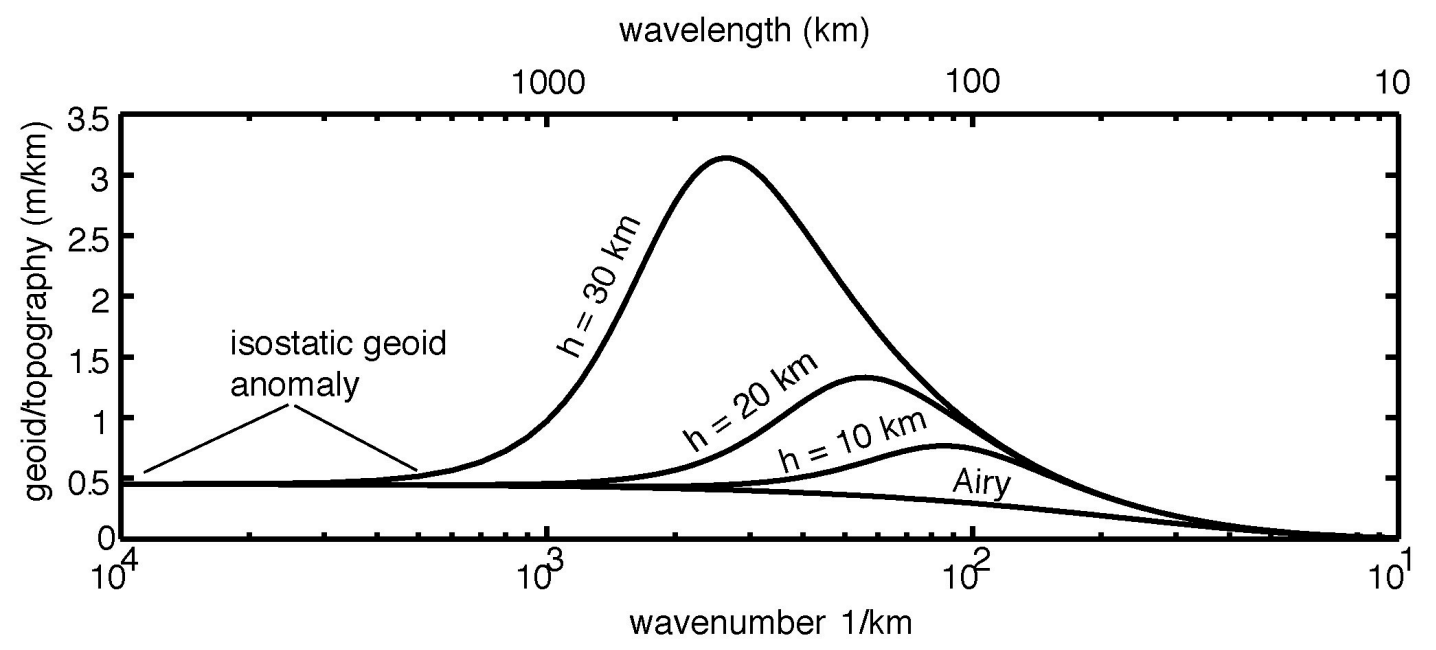

The amplitude of the geoid/topography transfer function is typically 0 to 4 meters per kilometer. This means that a seamount that is $1 \mathrm{~km}$ tall will produce a bump on the ocean surface that is about 1 meter tall. Since satellite altimeters have accuracy of better than $0.1 \mathrm{~m}$, such seamounts will be apparent in global geoid height maps. The shape of the geoid/topography transfer functions also depends on the thickness of the elastic plate.

There is one important difference between the geoid and the gravity anomaly. At long wavelengths, the gravity/topography transfer function goes to zero because the topography is 
isostatically compensated so the gravity signal from the topography is exactly cancelled by the gravity signature from the Moho. In contrast, the geoid/topography transfer function goes to a constant value at long wavelengths. We'll exploit this long-wavelength behavior in the next section to develop a very simple approach to constructing geoid height models.

\section{Isostatic Geoid Anomalies}

Section 5-12 of Geodynamics [2002] has a nice discussion of isostatic geoid anomalies including several of the more important applications of this approximation. However, their derivation is performed in the space domain and I think the wavenumber domain derivation is easier to understand and fits in with all of my previous gravity notes. In the lecture on Poisson's equation, we derived the formula for computing geoid height from an arbitrary 3-D density model $\Delta \rho(x, y, z)$.

$N(\mathbf{k})=\frac{G}{g} \int_{-\infty}^{o} \rho(\mathbf{k}, z) \frac{e^{2 \pi|\mathbf{k}| z}}{|\mathbf{k}|} d z$

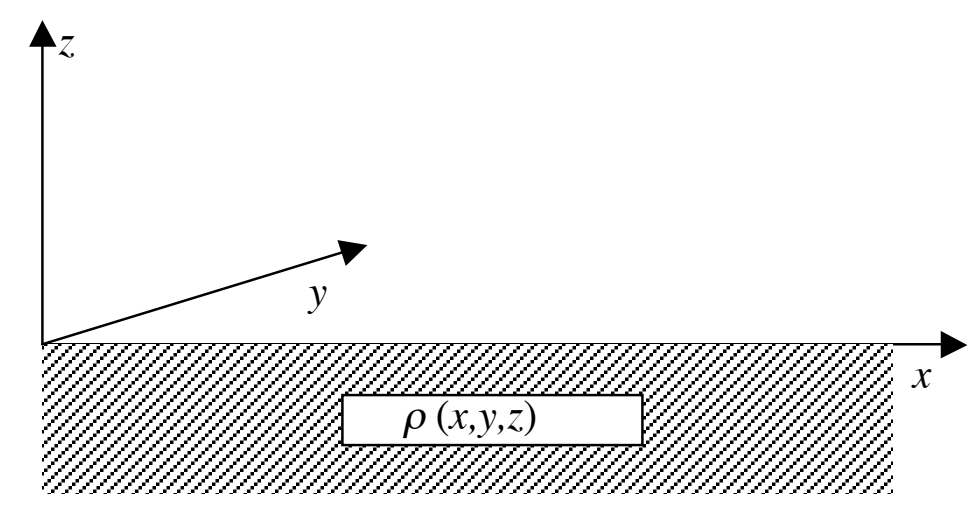

where $\Delta \rho(\mathbf{k}, z)=\mathfrak{S}^{2}[\Delta \rho(\mathbf{x}, z)]$. If the topography is isostatically-compensated, then

$0=\int_{-\infty}^{0} \Delta \rho(\mathbf{x}, z) d z=\int_{-\infty}^{0} \Delta \rho(\mathbf{k}, z) d z$

Now expand the exponential in equation 11 in a Taylor series about zero wavenumber. 
$N(\mathbf{k})=\frac{G}{g|\mathbf{k}|} \int_{-\infty}^{o} \Delta \rho(\mathbf{k}, z)\left[1+2 \pi|\mathbf{k}| z+\frac{(2 \pi|\mathbf{k}| z)^{2}}{2}+\cdots\right] d z$

Note that the first term in the brackets represents the integral of the density anomaly over depth and this is zero because of isostasy (equation 12). Next we assume that the wavelength of the anomaly is much greater than the depth of compensation $\lambda>>$ or $|\mathbf{k}| z<<1$. In this longwavelength limit, the third and higher-order terms are small compared with the second term. The integral simplifies to

$$
N(\mathbf{k})=\frac{2 \pi G}{g} \int_{-\infty}^{o} \Delta \rho(\mathbf{k}, z) z d z
$$

Now take the inverse fourier transform of (14).

$$
N(\mathbf{x})=\frac{2 \pi G}{g} \int_{-\infty}^{o} \Delta \rho(\mathbf{x}, z) z d z
$$

This is a remarkable results because this formula provides a way to compute the geoid height simply by integrating the density anomaly only over depth. This integration can be done for a variety of models including Airy compensation, Pratt compensation, and thermal compensation (i.e., spreading ridge or thermal swell). Several of these integrals are done in Geodynamics [2002].

\section{Isostatic Geoid and the Swell-Push Force}

The three driving forces of plate tectonics are slab pull, asthenospheric drag, and ridge push. Ridge-push force is the outward force due to isostatically-compensated topography. This mainly occurs as a gravitational sliding force on the flanks of the seafloor spreading ridges. However, it is also associated with the outward force due to thick continental crust or a thermal swell. One of your homework problems was to develop a general expression for this swell-push force for isostatically-compensated topography. Here I'll derive this expression again and point out that the force integral is identical to the integral for computing isostatic geoid anomaly. Therefore the two are related and one can use geoid height to measure ridge-push force [Parsons and Richter, 1980; Dahlen, 1981; Fleitout and Froidevaux, 1982; 1983].

Consider isostatically-compensated topography as shown in the diagram below.
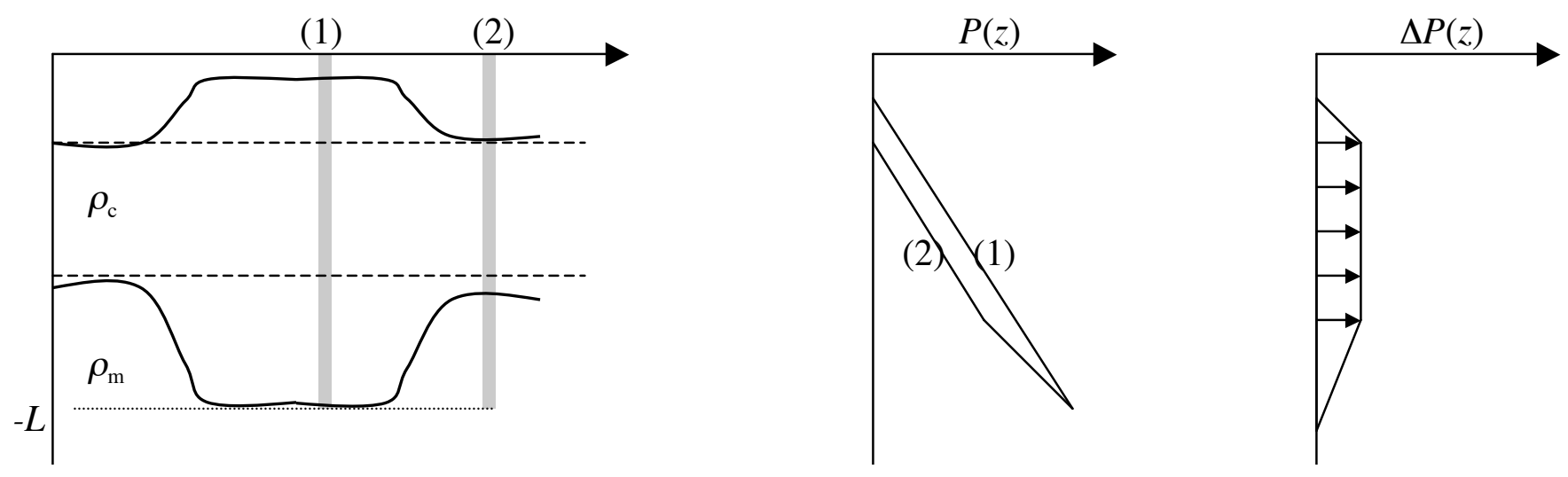
While this diagram is related to a specific Airy-type compensation mechanism, the integral relation is, in fact, quite general. To calculate the total outward force $F_{s}$ due to this isostaticallycompensated plateau, we integrate the difference in pressure between column (1) and column (2) over depth to the depth of compensation $-L$ (i.e. where the pressure difference is zero).

$$
F_{s}=\int_{-L}^{o} \Delta P(z) d z
$$

Integrate by parts

$$
F_{s}=\left.\Delta P(z)\right|_{-L} ^{0}-\int_{-L}^{o} \frac{\partial \Delta P(z)}{\partial z} z d z
$$

Note the first term on the right is zero because of isostasy. The second term can be written in terms of the density by noting that the vertical gradient in the pressure difference is

$$
\frac{\partial \Delta P(z)}{\partial z}=-\mathrm{g} \Delta \rho(\mathrm{z})
$$

The result is

$$
F_{s}=g \int_{-L}^{o} \Delta \rho(z) z d z
$$

Comparing equation 15 to equation 19, we see the integrals are the same so there is a direct relationship between swell-push force and geoid height.

$$
F_{s}=\frac{g^{2}}{2 \pi G} N
$$

We'll use this result later to calculate the ridge-push force versus age from the geoid height versus age relation.

\section{References}

Banks, R. J., R. L. Parker, and S. P. Huestis, Isostatic compensation on a continental scale: Local versus regional mechanisms, Geophys. J. R. astr. Soc., 51, 431-452, 1977.

Dalen, F. A., Isostasy and the ambient state of stress in the oceanic lithosphere, J. Geophys. Res., 86, 7801-7807, 1981.

Dorman, L. M., and B. T. R. Lewis, Experimantal isostasy 3: Inversion of the isostatic Green's function and lateral density changes, J. Geophys. Res., 77, 3068-3077, 1972.

Fleitout, L., and C. Froidevaux, Tectonics and topography for a lithosphere containing density heterogeneities, Tectonics, 1, 21-56, 1982. 
Fleitout, L., and C. Froidevaux, Tectonic stresses in the lithosphere, Tectonics, 2, 315-324, 1983. Haxby, W. F., and D. L. Turcotte, On isostatic geoid anomalies, J. Geophys. Res., 83, 5473$5478,1978$.

McNutt, M., Compensation of oceanic topography: An application of the response function technique to the Surveyor area, J. Geophys. Res., 84, 7589-7598, 1979.

McKenzie, D. P., Some remarks on heat flow and gravity anomalies, J. Geophys. Res., 72, 6261 6273, 1976.

Parsons, B., and F. M. Richter, A relationship between the driving force and geoid anomaly associated with mid-ocean ridges, Earth Planet. Sci. Lett., 51, 445-450, 1980.

Smith, W. H. F., and D. T. Sandwell, Bathymetric prediction fron dense satellite altimetry and sparse shipboard bathymetry, J. Geophys. Res., 99, 21803-21824, 1994.

Turcotte, D. L., and G. Schubert, Geodynamics: Second Edition. Cambridge, UK: Cambridge University Press, 2002. 